\title{
Forum
}

\section{Schwerpunktthema: Klimaethik - Ethische Implikationen des Klimawandels}

Das Thema Klima ist en vogue. Ausgehend von den naturwissenschaftlichen Diskussionen um den Klimawandel im letzten Viertel des 20. Jahrhunderts ist es nun bereits seit einigen Jahren auch in der Öffentlichkeit, den Medien und der Politik angekommen. Dies wird nicht zuletzt mit Blick auf die Vielzahl von Klimakonferenzen der letzten ca. 20 Jahre deutlich, an denen vermehrt hochrangige Politiker teilgenommen und miteinander um Perspektiven und Wege mit dem Ziel der Bekämpfung des Klimawandels gerungen haben.

Ethik erscheint in dieser Perspektive als ein Instrument modernen Politikmanagements. Doch weisen ,ethische Impulse“ der Klimadebatte wirklich auf einen neuen Politikstil hin und stehen sie für eine nachhaltige Korrektur eines bisher zu stark auf naturwissenschaftlich-technische Daten ausgerichteten Politikprozesses? Sorgen neue Perspektiven auf die gesellschaftlichen Aspekte des Klimawandels und innovative Organe eines Ethik-Managements im Bereich der Klimapolitik wirklich für bessere und gerechtere Entscheidungen? Ist „Klima-Governance“ nur ein neues Modewort oder verändert sich die globale Akteurslandschaft unter dem Einfluss der Debatten um die ethische Dimension des Klimawandels tatsächlich?

Das folgende Forum „Klimaethik“ thematisiert vor diesem Hintergrund aus dem Blickwinkel unterschiedlicher Teildisziplinen der Politikwissenschaft die ethischen Implikationen, die mit Fragen des Klimawandels und insbesondere seiner Bekämpfung verbunden sind. Marco Iorio erkennt dabei aus theoretischer Perspektive in Klimaethik eine neue Subdisziplin der Angewandten Ethik. Christoph Bieber beleuchtet aus dem Blickwinkel der Politischen Systemforschung die Einbettung klimaethischer Positionen in die politische Praxis am Beispiel der Ethikkommission für sichere Energieversorgung. Astrid Schulz und Bernd Sommer rücken schließlich die ethische Frage der Gerechtigkeit beim weltweiten Klimaschutz aus der internationalen Perspektive in den Mittelpunkt ihres Essays. 